\title{
Determination of the beam waist position for the spin-orbit interaction effect observation
}

\author{
E.A. Bibikova ${ }^{1,2}$, N.D. Kundikova ${ }^{1,2}$, A.A. Shulginov ${ }^{1}$, N. Al-Wassiti ${ }^{3}$ \\ ${ }^{1}$ South Ural State University, Chelyabinsk, Russian Federation; \\ ${ }^{2}$ Institute of Electrophysics, Ural Branch of the Russian Academy of Sciences, Ekaterinburg, Russian Federation; \\ ${ }^{3}$ Department of Physics, College of Science, Al-Mustansiriyah University, Baghdad, Iraq
}

\begin{abstract}
The spin angular momentum and the extrinsic orbital angular momentum of light are associated with the polarization of light and the light propagation trajectory, respectively. Those momenta are interdependent not only in an inhomogeneous or anisotropic medium but even in free space. This interaction is called the spin-orbit interaction of light. The effects of the spin-orbit interaction of light manifest themselves in a small transverse shift of the beam field longitudinal component from the beam propagation axis in the waist region under the circular polarization sign change. They can be observed both for Gaussian beams and for structured beams. The effects of the spinorbit interaction of light should be taken into account when nanophotonics devices are created, but the detailed investigation of the effect had not been performed yet due to the low intensity noise image of the beam waist. Precise measurements of the focal waist centerline are needed to determine the transverse shift of the beam field longitudinal component of the asymmetric converging beam's waist under the circular polarization sign change. We propose methods for determining the transverse and longitudinal positions of the beam waist. Computer image processing methods made it possible to obtain the value of the beam waist's transverse position with an accuracy of $0.1 \mu \mathrm{m}$. These methods will allow further testing of the shifts' theoretical predictions, the values of which are the order of $1 \mu \mathrm{m}$. The results obtained can also be used for laser processing of materials by polarized light and precise positioning of the beam's focal spot at a surface.

Keywords: spin-orbit interaction of light, angular momentum of photon, waist position, image processing.

Citation: Bibikova EA, Kundikova ND, Shulginov AA, Al-Wassiti N. Determination of the beam waist position for the spin-orbit interaction effect observation. Computer Optics 2021; 45(5): 661-666. DOI: 10.18287/2412-6179-CO-861.
\end{abstract}

\section{Introduction}

There are three types of angular momentum of a photon: spin, intrinsic orbital, and extrinsic orbital [1]. The spin angular momentum is associated with the polarization of light [2], the light beam trajectory determines the extrinsic orbital angular momentum (OAM) [3], and the intrinsic orbital angular momentum is determined by the light field's structure of the beam $[4,5]$.

The spin and extrinsic orbital angular momenta are interdependent in an inhomogeneous or anisotropic medium. This interaction is commonly called the spin-orbit interaction of light, also known as the optical Spin Hall Effect (an optical analog of the Spin Hall Effect that occurs for charge carriers in solid-state systems) $[6,7]$. In the most general case, the effect manifests itself in the transverse shift from the axis of propagation of the beam reflected from the two media boundary when the circular polarization sign changes. This shift is also known as the Imbert-Fedorov shift $[8,9,10]$. The transverse shifts at the core-cladding boundary result in the speckle pattern rotation of light propagating through the optical fiber under the circular polarization sign change, known as the Optical Magnus effect [11].

Such an effect can be observed in the free space, that is, regardless of the interaction of light with matter. Cur- rently, this effect is also known as the Geometric Optical Spin Hall effect. For the first time, the effect was predicted theoretically [12], namely, that the transverse shift of the "center of gravity" of the longitudinal component of the asymmetrically converging Gaussian beam occurs in the waist's plane under the sign of circular polarization change. The displacement is small, and its value is the order of the beam waist radius. In [13] the first visual results of the experimental detection of the waist shift were presented. For the same light propagation scheme, a generalized theory [14] was proposed, which applies to a beam with an arbitrary intensity distribution in the crosssection. The value of the "center of gravity" shift was calculated in the general case. It was also shown that the same shift of the "center of gravity" takes place in all sections of the converging beam, and not only in the plane of the waist. But in practice, the observation is possible just in the focal plane because of the large size of the beam compared to the small value of the shift. The paraxial approximation limits the accuracy of theoretical calculations in $[12,14]$.

Besides, the splitting of a collimated linearly polarized Gaussian beam into two (with left and right circular polarization) beams was found in the plane inclined to the direction of light propagation in the free space. This was 
theoretically demonstrated in [15]. The principal scheme of observation of this effect and experimental results were presented in $[16,17]$.

Further experimental investigations of the Geometric Spin Hall Effect were performed on the example of an asymmetric tightly focused beam. It consisted of two halves with the circular polarization of different signs in the cross-section [18]. In the waist's plane, the field's deformed distribution was observed due to the relative shifts of the electric energy density of the field's longitudinal components.

The intensity distribution of the $z$-component in the focal plane for a circularly polarized Gaussian beam is calculated [19]. The influence of the extrinsic orbital angular momentum on the spin angular momentum is experimentally demonstrated [20], that is, the influence of the trajectory of light propagation on its polarization in the free space. Several reviews have recently been published on spin-orbit interaction, generating light beams with angular moments, their registration, transformations, interactions with media, and applications of these phenomena $[21-23]$.

The effects of the spin-orbit interaction of light in the free space are weak [24, 25]. Methods of high sensitivity and accuracy are required to observe the effects of the spin-orbit interaction of light in the free space experimentally. Besides, it is necessary to apply modern image processing methods, allowing estimating small beam displacements of the order of $1 \mu \mathrm{m}$.

This work aims to propose the experimental methods for determining the Gaussian and the Bessel beam waist position and its displacement with high accuracy to observe the effects of the spin-orbital interaction of light subsequently. We should use low intensity and low contrast digital images with optical noise to solve this problem.

In general, the selection, recognition of objects, and determination of their parameters in the presence of various kinds of noises are some of the most complex and relevant tasks of image processing. In this case, the approximation problem arises since the original image has redundant information its use.

There are many algorithms for solving the approximation problem using the multivariable function [26], find edges [27-31], spline interpolation [32], including the use of adaptive triangular mesh [33, 34], and wavelets [35]. These algorithms are useful, for example, for compressing images or for recognizing their elements. However, to solve the problem, these methods are unsuitable, because the focal waist image has its features such as the lack of precise edges. It is not continuous but has a speckle structure. Therefore, an attempt to isolate the edges of the waist using differential operators leads to the appearance of impulse noises. Smoothing filters, tone correction, blurring, and contrasting algorithms cannot be used for the most precise determination of the waist parameters. Various types of threshold analysis, which are often used to highlight image elements and their edges, are also not recommended because all of these image processing methods add an unacceptable error.

Due to these limitations, the method of approximating the image by a two-dimensional function of many parameters using the least-squares algorithm was chosen, which involves fitting parameters that minimize the sum of the squared distances between each data point and the function. Theoretical assumptions regarding the beam properties allowed us to choose the function type and the function parameters.

\section{Method of determination of the Gaussian beam waist transversal position}

To determine the transverse position of the longitudinal component of the electrical field ( $z$-polarization) of the asymmetrically converging Gaussian beam in the waist region, we used the experimental setup shown in fig. 1. We observed the waist in the longitudinal direction (Oyz plane).

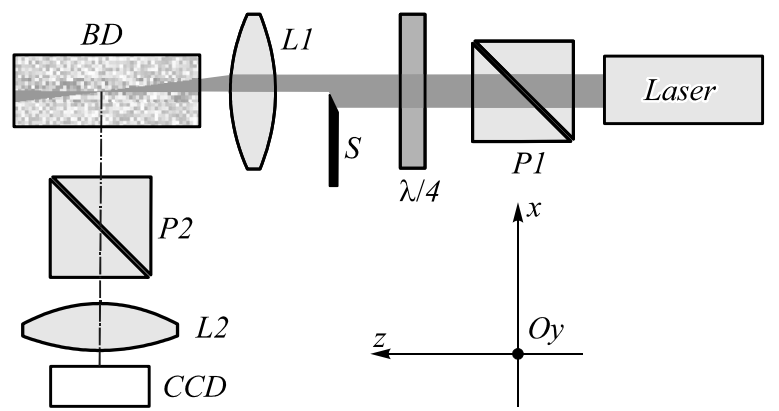

Fig. 1. The experimental setup for observation of the focal waist position of the Gaussian beam. P1, P2 are polarizes; $S$ is shutter; L1, L2 are lenses; $B D$ is beam diffuser

The He-Ne laser's radiation was passed through the beam expander, which enlarged the beam diameter by 2.54 times. The polarizer $\mathrm{P} 1$ and the phase plate $\lambda / 4$ were used to give circular polarization to the beam. Then, we got the beam's image in the transverse direction (Oxy plane) to determine its main parameters. The transverse image of the Gaussian beam is shown in fig. 2.

The intensity distribution of the beam cross-section shown in fig. 2 was approximated by the function:

$$
f(x, y)=a \cdot \exp \left(-\frac{x^{2}+y^{2}}{2 \sigma^{2}}\right)+f_{0} .
$$

The approximation of the image is shown in fig. 3. The diameter $d=2 \sigma$ of the beam by criteria $\mathrm{FWe}^{-2} \mathrm{M}$ was $2.30 \pm 0.01 \mathrm{~mm}$.

The expanded laser beam with circular polarization fell on shutter $\mathrm{S}$, which blocked half of the radiation. The lens L1 with the focal length of $20 \mathrm{~mm}$ formed the asymmetrically converged beam. The longitudinal image of the $z$-polarization of the beam is shown in fig. 4 . The image enlarged with the lens system L2 was obtained using a CCD camera. We installed a polarizer P2 in front of the camera to select the $z$-polarization of scattered light. 


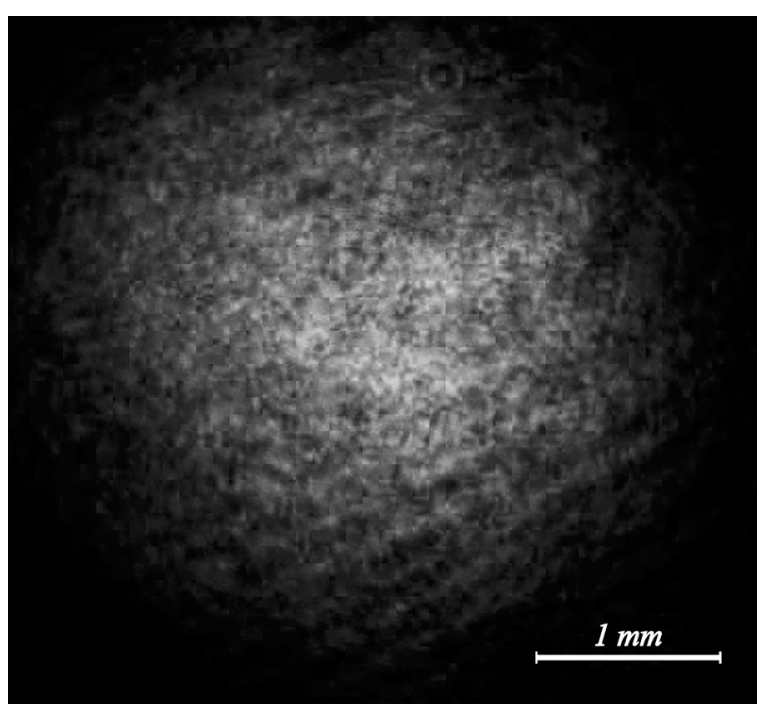

Fig. 2. The transverse image of the laser beam

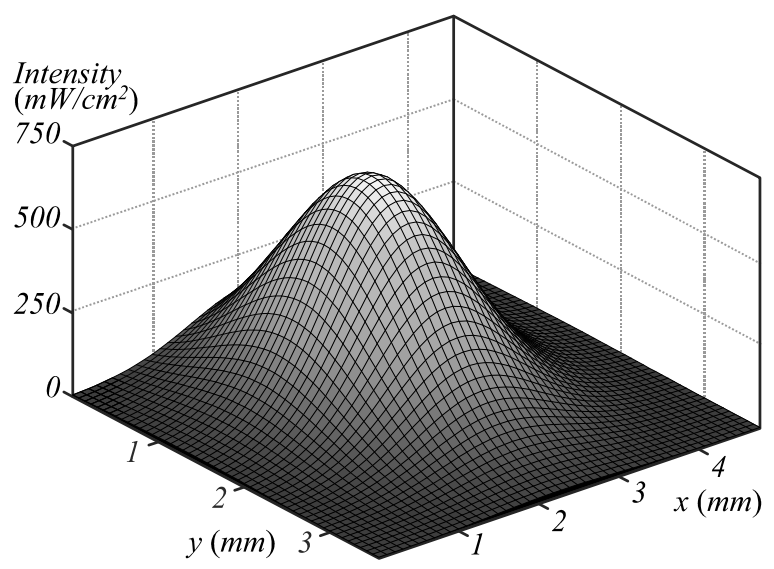

Fig. 3. The approximation of the Gaussian beam in the transverse direction

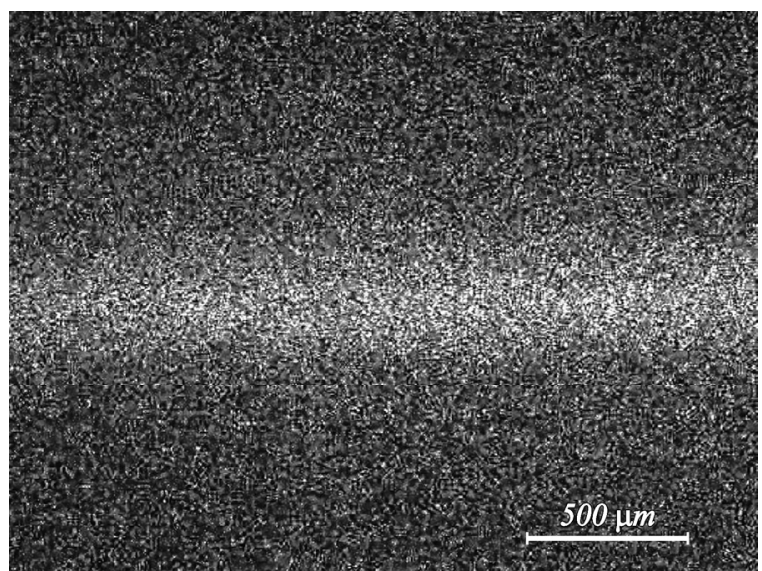

Fig. 4. Longitudinal image of the focal waist obtained by scattering the z-polarization of the Gaussian beam

A two-dimensional function approximated the image to determine the centerline of the beam in the longitudinal direction:

$$
\begin{aligned}
& f(y, z)=a(z) \cdot \exp \left(-\frac{\left(y-y_{0}-k z\right)^{2}}{2 \sigma^{2}(z)}\right)+f_{0}(y, z), \\
& a(z)=a_{0}+a_{1} z+a_{2} z^{2}, \sigma(z)=\sigma_{0}+k_{\sigma 1} z+k_{\sigma 2} z^{2},
\end{aligned}
$$

$$
f_{0}(y, z)=f_{1}+k_{z} z+k_{y} y .
$$

The intensity maximum change alone the axis $\mathrm{O} z$ (parameters $a_{0}, a_{1}, a_{2}$ ), width change (parameters $\sigma_{0}, k_{\sigma 1}$, $k_{\sigma 2}$ ), beam tilt, and position (parameters $y_{0}$ и $k$ ) were taken into account in the approximation function. The speckled inhomogeneous image background was also taken into account (parameters $f_{0}, k_{z}, k_{y}$ ). The variation was made on these 11 parameters. The image approximation of the longitudinal intensity distribution of the beam waist by Eq. 2 is shown in fig. 5 .

The bright background in the image (fig. 4) is due to the re-scattering of the radiation. That is why the contrast of the waist image is low. Nevertheless, the image approximation by Eq. 2 allowed us to determine the position of the centerline, the width, and the tilt of the beam quite precisely. In our experiments, the error of the transversal position of the centerline was $\sim 0.1 \mu \mathrm{m}$.

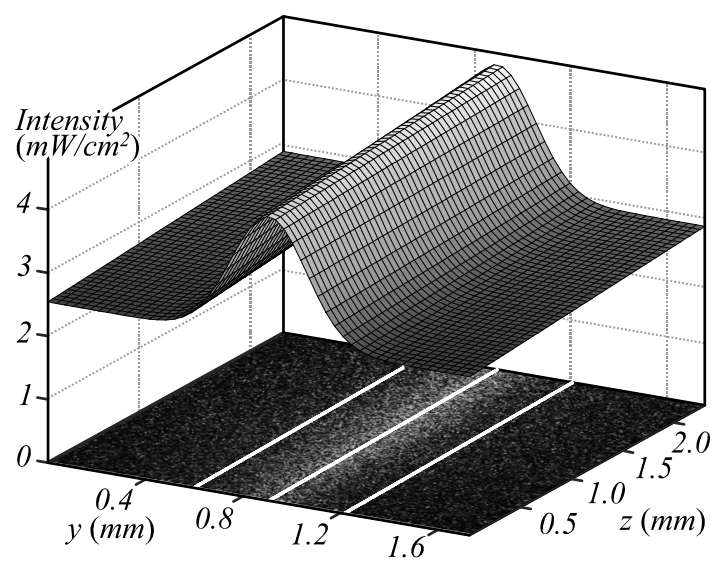

Fig. 5. The approximation of the z-polarization of the Gaussian beam waist image

\section{Method of determination of the Bessel beam waist transversal position}

To determine the transverse position of different electric field components ( $z$ - and $y$-polarization) of the asymmetrically converging Bessel beam (beam with nonzero intrinsic OAM) in the waist region, we used the experimental setup shown in fig. 6 . To create a beam with nonzero intrinsic OAM, an enlarged Gaussian beam was passed through the diffractive optical element (DOE). The topological charge $l$ of that beam was equal 2 . Such a beam with a spiral wavefront we conditionally called the Bessel beam. On passing the beam through DOE, the intensity distribution in the transverse direction is shown in fig. 7. The CCD camera was fixed after the phase plate $\lambda / 4$. The image was approximated by the function:

$$
f(x, y)=a \cdot J_{l}^{2}\left(\frac{\sqrt{x^{2}+y^{2}}}{R}\right)+f_{0},
$$

where $J_{l}(r)$ is the $l$-order first kind Bessel function.

This function takes zero value in $r=5.14$ for $l=2$. Therefore, the radius of the first dark ring equals $r_{1}=5.14 R$. Furthermore, the beam passed through the 
diaphragm D. Its radius was chosen to cut the central ring only.

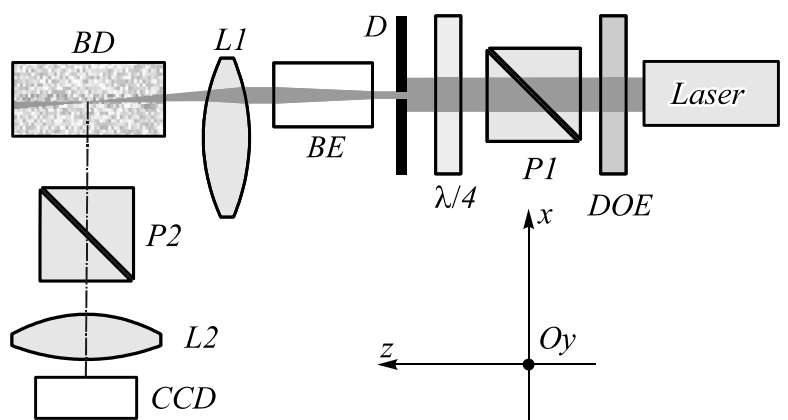

Fig. 6. The experimental setup for observation of the focal waist position of the beam with nonzero OAM. DOE is diffractive optical element; $P 1, P 2$ are polarizes; $D$ is diaphragm; $L 1, L 2$ are lenses; $B E$ is beam expander; $B D$ is beam diffuser

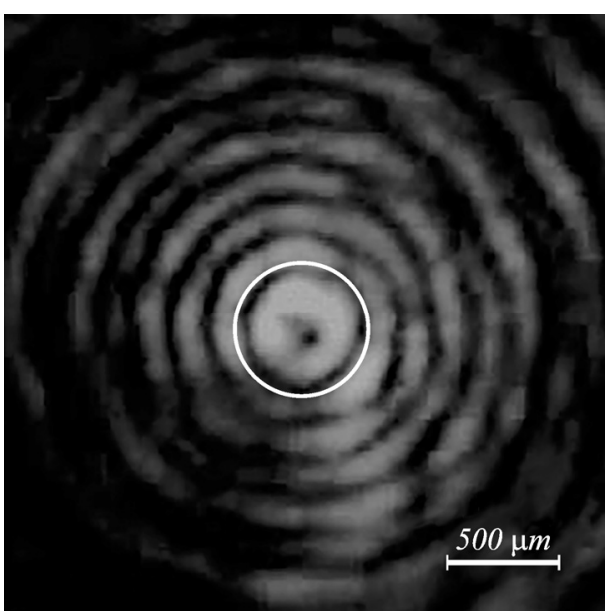

Fig. 7. The image of the Bessel beam in the transverse direction

An approximation of the transverse image of this part of the beam using Eq. 3 is shown in fig. 8 .

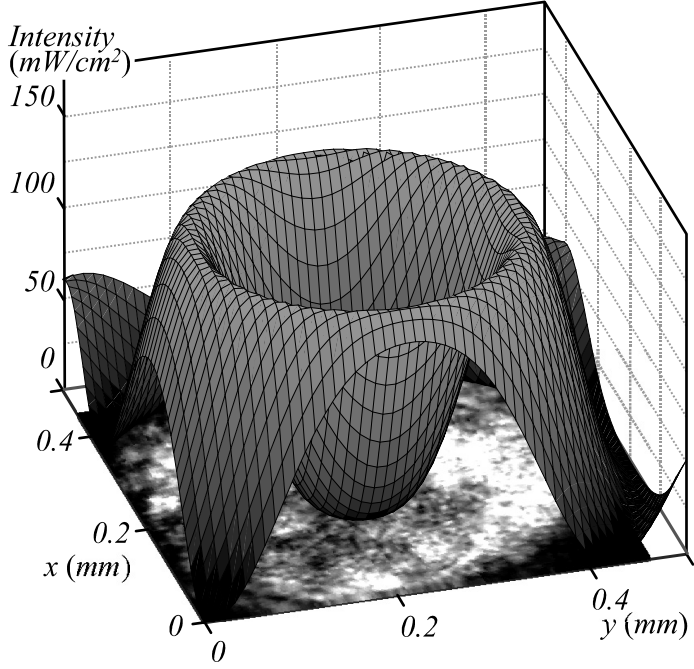

Fig. 8. The approximation of the Bessel beam in the transverse direction

The Bessel beam's central ring passed through the beam expander $(\mathrm{BE})$. The $\mathrm{BE}$ enlarged the ring diameter by 12 times. The enlarged beam passed through the upper half of lens L1 to form an asymmetrically converged beam.
The system for registration of the scattered light was the same as in the experiment with Gaussian beam (fig. 1).

The longitudinal image of the $y$-polarization of the Bessel beam is shown in fig. 9. This image shows the intensity decrease in the middle of the waist in the longitudinal direction. Therefore, approximating this image by a Gaussian function will be incorrect. The intensity distribution in the beam waist was approximated by the function (4):

$$
\begin{aligned}
& f(y, z)=a(z) \cdot F\left(\frac{y-y_{0}-k z}{s}\right)+f_{0}(y, z) \\
& a(z)=a_{0}+a_{1} z+a_{2} z^{2}, \\
& f_{0}(y, z)=f_{1}+k_{z} z+k_{y} y+k_{z 2} z^{2}+k_{y 2} y^{2}+k_{y z} y z, \\
& F(y)=\int_{-\xi}^{+\xi} J_{l}^{2}\left(\sqrt{x^{2}+y^{2}}\right) d x,-r \leq y \leq r, \\
& \xi=\sqrt{r^{2}-y^{2}} .
\end{aligned}
$$

The primary function is $F(y)$, in which the Bessel intensity distribution in the beam cross-section is taken into account. This function characterizes the scattered Bessel beam's projection onto the $\mathrm{O} y z$ plane, in which the CCD camera was placed. Secondary scattering in the medium was not taken into account, $y_{0}$ is the position of the central line of the waist in the transverse direction, and $s$ is its width. The coefficient $k$ depends on the tilt of the waist image. The difference in intensity along the waist $\left(a_{0}, a_{1}, a_{2}\right)$, and the background irregularity $\left(f_{1}, k_{z}, k_{y}, k_{z 2}, k_{y 2}, k_{y z}\right)$ were taken into account in Eq. 4 also. Equation 4 has 12 parameters, by which the variation was made. The result of image approximation (fig. 9) by this function is shown in fig. 10 .

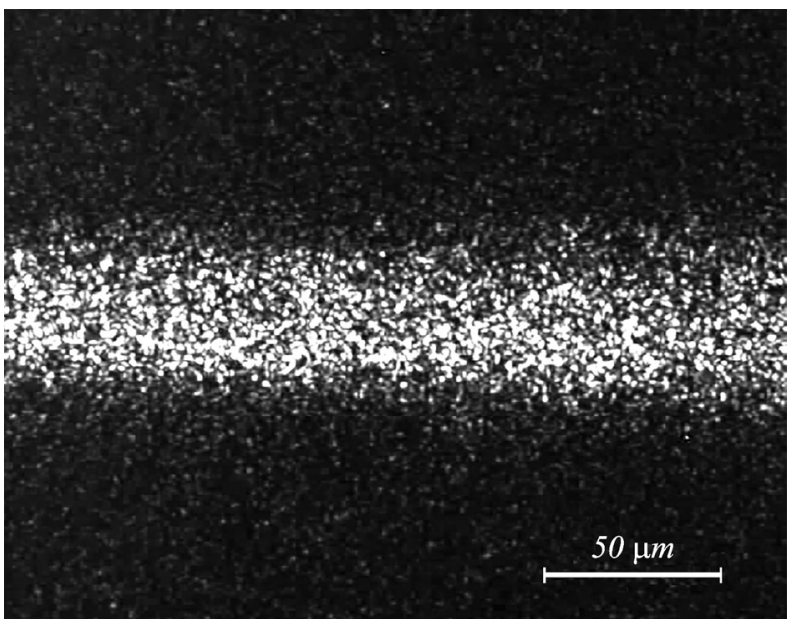

Fig. 9. The image of the y-polarization of the Bessel beam waist

The most impressive is the image processing the $z$ polarization of the Bessel beam waist. According to the theoretical predictions, namely, this component image could demonstrate the effect of spin-orbital interaction. However, a difficulty arises from the low intensity and low contrast of this image (fig. 11). Nevertheless, the approximation of this image by Eq. (4) allows us to determine the centerline of the Bessel's beam waist in the transverse di- 
rection (along the axis $\mathrm{O} y$ ) with accuracy $\sim 0.1 \mu \mathrm{m}$. Besides, such an approximation allows us to determine its width and tilt of Bessel beam waist (fig. 12).

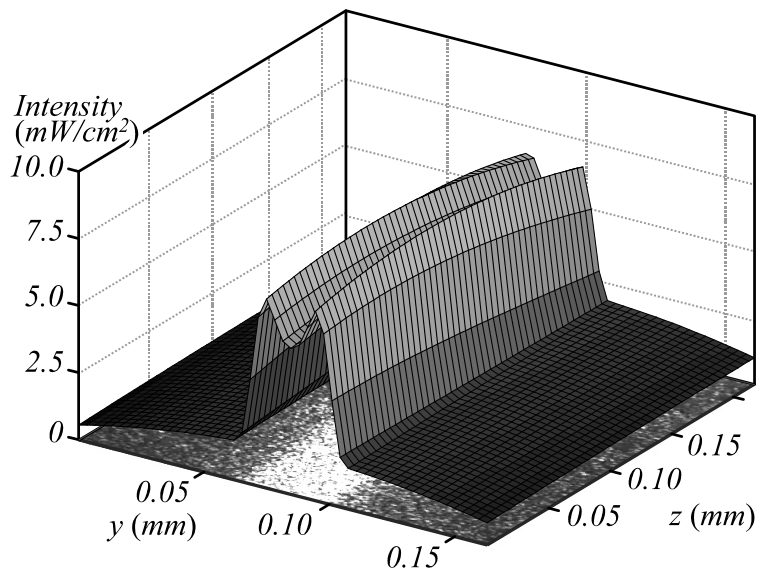

Fig. 10. The approximation of the y-polarization of the Bessel beam waist

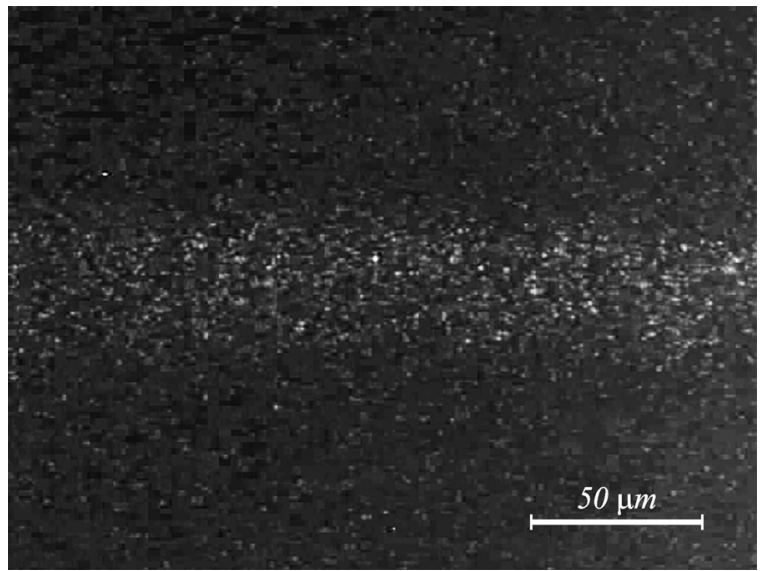

Fig. 11. The image of the z-polarization of the Bessel beam waist

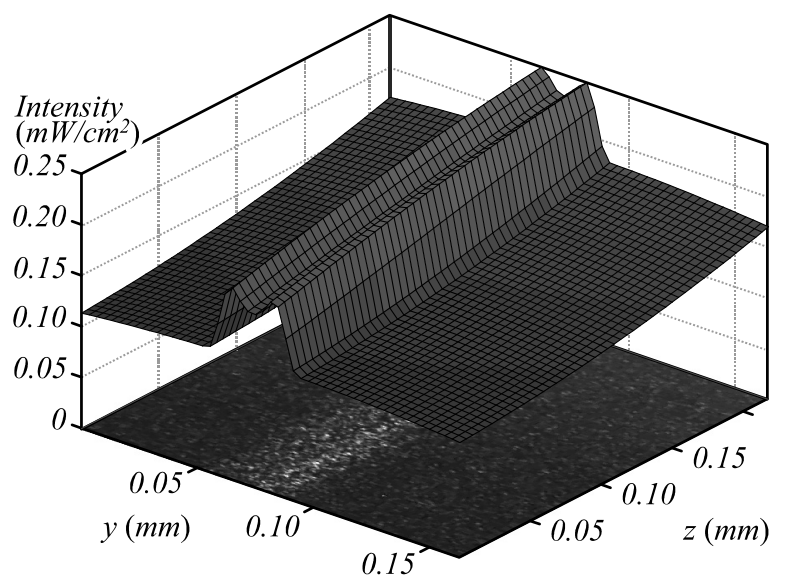

Fig. 12. The approximation of the z-polarization of the Bessel beam waist

We have demonstrated the effectiveness of the developed method for determining the shift of the waist of an asymmetric converging Gaussian beam under the circular polarization sign change. Fig. 13 shows the intensity distribution of the $z$ components of the left and right circular polarized Gaussian beam in the waist region. The devel- oped method has allowed us to detect the waist shift by the value $0.4 \pm 0.1 \mu \mathrm{m}$. We should emphasize that the image's shift was recorded visually at the first experimental observation of the effect. The waist position was determined by the lines drawn in the photographs $[13,36]$; the shift value was approximately $1.5 \mu \mathrm{m}$. Thus, the developed method will allow us to study the waist shift effect for different light beams in detail.

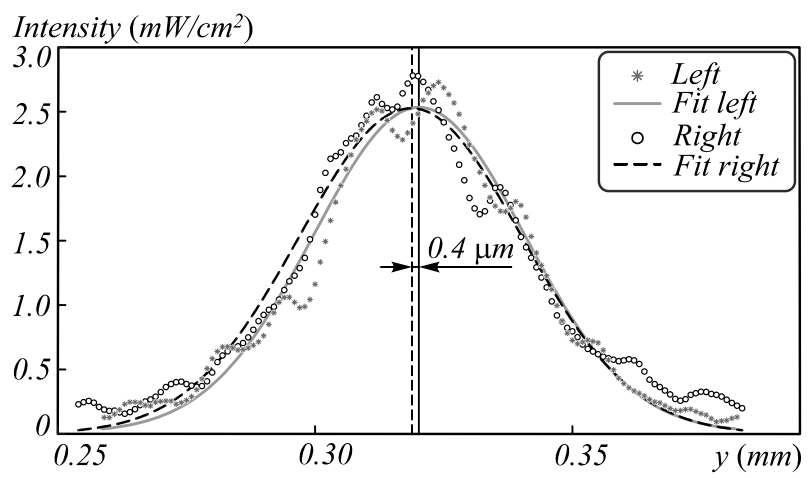

Fig. 13. The intensity distribution of the z-polarization in the beam waist and approximation by a Gaussian function for left and right circular polarization

\section{Conclusion}

We have proposed approximating noisy images of low intensity and low contrast without clear boundaries by special functions. The method turned out to be very effective for determining the beam's waist main characteristics (centerline position, width, tilt). We were successful in determining the beam waist longitudinal component's position with an accuracy of $0.1 \mu \mathrm{m}$. The developed method can be useful for studying the fine effects of the spin-orbit interaction of light in beams with different phase and intensity structures.

\section{References}

[1] Abdulkareem S, Kundikova N. Joint effect of polarization and the propagation path of a light beam on its intrinsic structure. Opt Express 2016; 24: 19157-19165.

[2] Beth RA Mechanical detection and measurement of the angular momentum of light. Phys Rev 1936; 50: 115-125.

[3] Landau LD, Lifshitz EM, Berestetskii VB, Pitaevskii LP. Course of Theoretical Physics. 4: Quantum Electrodynamics. $2^{\text {nd }}$ ed. Pergamon Press Ltd; 1982.

[4] Allen L, Barnett SM, Padgett MJ. Optical angular momentum. Bristol: Institute of Physics; 2003.

[5] Bliokh K. Geometrical optics of beams with vortices: Berry phase and orbital angular momentum Hall effect. Phys Rev Lett 2006; 97: 043901.

[6] Onoda M, Murakami S, Nagaosa N. Hall effect of light. Phys Rev Lett 2004; 93: 083901.

[7] Bliokh K, Bliokh Y. Conservation of angular momentum, transverse shift, and spin Hall effect in reflection and refraction of an electromagnetic wave packet. Phys Rev Lett 2006; 96: 073903.

[8] Fedorov FI. On the theory of total internal reflection. Dokl Akad Nauk SSSR 1955; 105(5): 465-469.

[9] Imbert C. Experimental proof of the photon's translational inertial spin effect. Phys Lett A 1970; 31: 337-338. 
[10] Bliokh K, Aiello A. Goos-Hänchen and Imbert-Fedorov beam shifts: an overview. J Opt 2013; 15: 014001.

[11] Dooghin AV, Kundikova ND, Liberman VS, Zeldovich BYa. Optical Magnus effect. Phys Rev A 1992; 45: 8204-8208.

[12] Baranova NB, Savchenko AYa, Zel'dovich BYa. Transverse shift of a focal spot due to switching of the sign of circular polarization. JETP Lett 1994; 59(4): 232-234.

[13] Kundikova ND, Podgornov FV, Rogacheva LF, Zel'dovich BYa. Manifestation of spin-orbit interaction of photon in a vacuum. Pure Appl Opt 1995; 4: 179-183.

[14] Bekshaev A, Bliokh KY, Soskin M. Internal flows and energy circulation in light beams. J Opt 2011; 13: 53001.

[15] Aiello A, Lindlein N, Marquardt C, Leuchs G. Transverse angular momentum and geometric spin hall effect of light. Phys Rev Lett 2009; 103: 100401.

[16] Korger J, Aiello A, Gabriel C, Banzer P, Kolb T, Marquardt C, Leuchs G. Geometric Spin Hall Effect of Light at polarizing interfaces. Appl Phys B 2011; 102: 427.

[17] Korger J, Aiello A, Chille V, Banzer P, Wittmann C, Lindlein $\mathrm{N}$, Marquardt C, Leuchs G. Observation of the geometric spin Hall effect of light. Phys Rev Lett 2014; 112: 113902.

[18] Neugebauer M, Banzer P, Bauer T, Orlov S, Lindlein N, Aiello A, Leuchs G. Geometric spin Hall effect of light in tightly focused polarization tailored light beams. Phys Rev Lett 2014; 89: 013840.

[19] Zhao Y, Edgar JS, Jeffries GDM, McGloin D, Chiu DT. Spin-to-orbital angular momentum conversion in a strongly focused optical beam. Phys Rev Lett 2007; 99: 073901.

[20] Bibibkova, E.A. Kundikova, N.D. Rogacheva, L.F. "Influence of the light trajectory on the light polarization in an optically homogeneous medium," Proceedings of the Chelyabinsk Scientific Center, N. 3, 2006, pp. 91-95.

[21] Fu S, et al. Spin-orbit optical Hall effect. Phys Rev Lett 2019; 123(24): 243904.

[22] Zhu L, Wang J. A review of multiple optical vortices generation: methods and applications. Front Optoelectron 2019; 12(1): 52-68.

[23] Shen Y, et al. Optical vortices 30 years on: OAM manipulation from topological charge to multiple singularities. Light Sci Appl 2019; 8(1): 90.
[24] Li J, Zhang J, Li J. Optical twists and transverse focal shift in a strongly focused, circularly polarized vortex field. Opt Commun 2019; 439(February): 284-289.

[25] Kotlyar VV, Stafeev SS, Kovalev AA. Sharp focusing of a light field with polarizationand phase singularities of an arbitrary order. Computer Optics 2019; 43(3): 337-346. DOI: $10.18287 / 2412-6179-2019-43-3-337-346$.

[26] Iske A. Approximation theory and algorithms for data analysis. Cham: Springer Nature Switzerland AG; 2018.

[27] Nayak DR, Sahu SK, Mohammed J. A cellular automata based optimal edge detection technique using twentyfive neighborhood model. Int J Comput Appl 2013; 84(10): 27-33.

[28] Balabantaray BK, Sahu OP, Mishra N. A quantitative performance analysis of edge detectors with hybrid edge detector. J Comput 2017; 12(2): 165-173.

[29] Belim SV, Kutlunin PE. Boundary extraction in images using a clustering algorithm. Computer Optics 2015; 39(1): 119-124. DOI: 10.18287/0134-2452-2015-39-1-119-124.

[30] Shulginov AA, Stadnik OS. Recognition and classification of plasma clots of bioelectograms. Proc 2018 Global Smart Industry Conference (GloSIC) 2018: 1-5.

[31] Bettaieb A, Filali N, Filali T, Ben Aissia H. GPU acceleration of edge detection algorithm based on local varianceand integral image: application to air bubbles boundaries extraction. Computer Optics 2019; 43(3): 446-454. DOI: 10.18287/2412-6179-2019-43-3-446-454

[32] Schumaker LL. Spline functions: Computational methods. SIAM; 2015.

[33] Demaret L, Iske A. Anisotropic triangulation methods in adaptive image approximation. In Book: Georgoulis E, Iske A, Levesley J, eds. Approximation algorithms for complex systems. Berlin, Heidelberg: Springer; 2009: 47-68.

[34] Li X. Anisotropic mesh adaptation for image representation. EURASIP J Image Video Process 2016; 1: 26.

[35] Dremin IM, Ivanov OV, Nechitailo VA. Wavelets and their uses. Physics-Uspekhi 2001; 44(5): 447-478.

[36] Zel'dovich BYa, Kundikova ND, Rogacheva LF. Observed transverse shift of a focal spot upon a change in the sign of circular polarization. JETP Lett 1994; 59: 766-769.

\section{Authors' information}

Evelina Anatolyevna Bibikova (b. 1980), graduated from South Ural State University in 2003. She received her Candidate's degree in Physics and Mathematics from Institute of Electrophysics in 2007 in the field of Optics. She currently works as a researcher in the Nonlinear Optics Laboratory of Institute of Electrophysics and as an associate professor at South Ural State University. E-mail: bibikovaea@susu.ru.

Nataliya Dmitrievna Kundikova (b. 1952), graduated from Moscow State University in 1975. She received her Doctor's degree in Physics and Mathematics from Moscow State University in 1996 in the field of Optics. She currently works as a head of the Nonlinear Optics Laboratory of Institute of Electrophysics and a dean of the Physics department of South Ural State University. E-mail: kundikovand@susu.ru.

Alexander Anatolyevich Shulginov (b. 1963), graduated from Moscow Institute of Physics and Technology in 1987. He received his Candidate's degree in Physics and Mathematics in 1994 in the field of Optics. He currently works as an associate professor at South Ural State University. E-mail: shulginovaa@susu.ru.

Nazar Al-Wassiti (b. 1973), graduated from University of Baghdad in 2002. He currently works as an assistant teacher at Mustansiriyah University. E-mail: nazarjabir@gmail.com .

Code of State Categories Scientific and Technical Information (in Russian - GRNTI): 29.03.45; 29.03.31 Received January 13, 2021. The final version - April 30, 2021. 